rienced by some old people after cataract operations, when, as Trevor-Roper ${ }^{1}$ said, the pebble-lenses needed after the operation surround them by a distorted world which squirms about with every eye movement, while "faces pop in and out of the blind area with the annoying insolence of a jack-in-thebox." He believes that many a frail psyche faced with the choice would prefer "to grope its way through life rather than cope with this formidable new visual world." The hemiplegic patient does not have any choice, and the "frail psyche" many are left with needs all possible support and consideration to make life tolerable.

Patients isolated by deafness sometimes become introspective, withdrawn, depressed, or even delusional. Their inaccessibility may make heavy demands on the patience of those who are trying to help them. It should be remembered that distortion of the quality of sound and intolerance of amplification are common in perceptive deafness, and patients should not be shouted at; instead they must be addressed in clear, explicit speech, from a position which permits them to lipread.

The intimate association between sensation and motor function is well known. Defective proprioception denies the patient an assured knowledge of joint position and movement, disrupts the feed-back mechanisms maintaining muscle length and tension, and upsets the control of balance, control of the arm, and control of walking. Disordered discriminative sensation at cortical level interferes with touch localization and awareness of shape, size, form, and texture. This has distinctive effects on the precision use of the hand and fingers, contributing perhaps to forms of apraxia. Impaired cortical integration upsets consciousness of body image, creating problems of neglect of the hemiplegic limbs (despite effective recovery of voluntary power) or even more bizarre symptoms, such as loss of awareness of paralysis or even denial of ownership of limbs.

Clinical patterns of disordered sensation are variable not only from one patient to another but also in the same patient from time to time, when levels of awareness and atten- tiveness are patchy. This may account for variable levels of performance, and adds to the difficulties of prognosis in the early stages of recovery.

\section{POSTURAL CONTROI}

The patient who cannot sit up with confidence cannot stand, and the patient who cannot stand reliably cannot walk.

There are two pathways of postural control which may be affected in cerebrovascular accidents, either vestibular/cerebellar co-ordination in the brain stem or the visuo-spatial integrating systems of the parietal cortex. These sensitive mechanisms are probably dependent on a complex network of intracerebral and basal ganglial connexions, and it is not surprising that some disorder of postural fixation and balance, temporary or more persistent, is commonplace in recovery from strokes.

\section{Conclusion}

Use of the foregoing criteria sets a pattern of assessment of capacity after a stroke which can indicate when to treat, what to treat, and how to treat it, matching the management of the patient with progress past the milestones of recovery from sitting to standing, then to walking, and finally to social reliability. In the early years, before this systematic follow-up was applied to treatment, about equal numbers of the patients who survived severe or moderately severe strokes recovered or became long-stay failures. Results now show a $30 \%$ difference in favour of recovery, ${ }^{2}$ a tribute to the more imaginative and constructive efforts being made to overcome these mental barriers to recovery from strokes.

\section{References}

1 Trevor-Roper, P. D., British Medical fournal, 1970, 3, 33.

2 Adams, G. F., Gerontologia Clinica. In press.

\title{
Coronary Artery Disease
}

\section{I-Management of the Acute Episode}

\section{B. L. PENTECOST}

British Medical fournal, 1971, 1, 93-95

The current treatment of acute myocardial infarction is designed to deal with two well-recognized features of its natural history: firstly, a peak mortality within the first few hours of the episode ${ }^{2}$; and, secondly, a high incidence of deaths related to arrhythmias rather than to massive injury of the myocardium. There has, therefore, been a general trend

The General Hospital, Birmingham 4

B. L. PENTECOST, M.D., M.R.C.P., Consultant Physician

towards early hospital admission, particularly in the younger good-risk patient. Conversely, in an elderly patient with recurrence of infarction together with pre-existing chronic failure the main problems are likely to be those of severe mechanical failure. In this case home treatment may under certain conditions be preferable to hospital care. The early occurrence of arrhythmias reduces the therapeutic potential of the hospital coronary care unit and has led to the development of mobile units in some centres. ${ }^{34}$ The achievements of mobile and hospital units alike have been mainly in treating arrhythmias rather than those complications resulting from severe myocardial injury. 


\section{General Care}

Frequently the patient's first need is for effective analgesia. Diamorphine (heroin) is a rapidly acting analgesic largely free from the side effects of nausea and vomiting ${ }^{5}$ and the rarer syndrome of bradycardia and hypotension ${ }^{6}$ associated with morphine. ${ }^{7}$ Pentazocine given parenterally in a doses of $30 \mathrm{mg}$ is a useful alternative and certainly does not depress systemic arterial pressure or respiration ${ }^{89}$ as does the morphine group. It does, however, appear to be slower in action than diamorphine $^{8}$ and a report of a rise in pulmonary arterial pressure following its administration ${ }^{10}$ has served to diminish enthusiasm for the drug.

Though there are certain haemodynamic advantages from being nursed in the propped up position ${ }^{11}$ the optimal nursing posture varies greatly. A man in left ventricular failure is more comfortable sitting bolt upright, whereas a hypotensive patient is obviously better off supine. The duration of rest in bed depends on individual progress but the patient, who has an uncomplicated course may be sitting out of bed within the week, walking within ten days, and home soon after two weeks.

Routine administration of oxygen is a time-honoured aspect of general care in myocardial infarction. It is probably justified on the grounds that the arterial $\mathrm{PO}_{2}$ is commonly reduced after infarction ${ }^{12}$ and, in the absence of shock, readily corrected by conventional oxygen therapy. It is hoped that raising the oxygen content of the tissue immediately surrounding the infarction may limit the final extent of myocardial injury. ${ }^{13}$

The value of anticoagulant therapy remains debatable, though it does appear to reduce the incidence of thromboembolic complications. Many doctors have ceased to use anticoagulant drugs routinely, rather seeking to reduce venous thrombosis and pulmonary embolism by getting the patient up early. It is my practice to give anticoagulant drugs only to patients who are for some reason unusually immobile-for example, following multiple episodes of cardiac arrest.

\section{Ventricular Arrhythmias}

Sudden death in the first few hours after myocardial infarction is usually the result of ventricular fibrillation. Prompt resuscitative procedures have led to the correction of this arrhythmia in many patients, both in hospital and outside, with subsequent discharge of the patient back to his former activities.

In the coronary care unit successful restoration of normal heart rhythm should be achieved in about $90 \%$ of patients in whom the fibrillation occurs in the absence of myocardial failure-the so-called primary ventricular fibrillation. Of these patients, some three-quarters may be expected to return home from hospital. ${ }^{14}{ }^{15}$ The chance of producing a survivor with severe intellectual impairment appears to be negligible. Patients with evidence of severe brain damage seldom survive initial resuscitation for very long.

The chances of successful resuscitation are increased by immediate defibrillation-by doctor or nurse-without an intervening period of cardiac massage and artificial ventilation. It should be possible to achieve this in a coronary care unit, though initial supportive measures are inevitably needed when the arrest occurs elsewhere. Encouragingly, patients who have survived ventricular fibrillation enjoy a similar long-term prognosis to those who have not had this complication. $^{16}{ }^{17}$

After resuscitation ventilatory function must be assessed with care. Patients occasionally require artificial ventilation before adequate spontaneous ventilation is re-established. Rarely the paradoxical movements of flail chest may be seen after a period of external massage; under these circumstances assisted ventilation will be required for a longer period.

In contrast, cardiac arrest which complicates pulmonary oedema or the shock syndrome is frequently fatal. Normal rhythm may occasionally be restored but there is frequently no mechanical counterpart.

\section{ANTIARRHYTHMIC THERAPY}

Routine antiarrhythmic therapy is usually administered after ventricular fibrillation to try to prevent further episodes of cardiac arrest. Intravenous lignocaine $1-3 \mathrm{mg} / \mathrm{min}$. is given by continuous infusion. The required dose is difficult to gauge in the absence of an arrhythmia-for example, ventricular extrasystoles-the suppression of which provides a useful indicator, but a dose of $2 \mathrm{mg} / \mathrm{min}$. probably provides a therapeutic effect. ${ }^{18}$ Lignocaine is not free from side effects, and if the infusion is poorly controlled an overdose may result in the appearance of hypotension, aggravation of heart failure, or mental confusion and even coma.

We have found that recurrent ventricular fibrillation which occurs despite drug therapy is best treated by suppression of ventricular ectopic activity by pacing the heart from the right ventricle. ${ }^{19}$ Though rates of up to $140 / \mathrm{min}$. may be required initially to control frequent ventricular premature beats and bursts of ventricular tachycardia, usually the rate can be reduced to just above the original one within 30 minutes. The cardiac catheter may also be used to terminate ventricular tachycardia, again by rapid pacing, though not, of course, ventricular fibrillation.

\section{PREVENTION OF CARDIAC ARREST}

Recently emphasis has swung towards prevention of cardiac arrest. The presence of frequent ventricular extrasystoles or premature beats, particularly when they occur in salvos, or when superimposed upon the $T$ wave of the preceding $Q R S$ complex are known to provoke fibrillation. It has been claimed that the aggressive therapy of such warning arrhythmias reduces the incidence of arrest. ${ }^{20}$ This is not to say that every ventricular premature beat-an almost universal accompaniment of acute infarction-invites the doctor to bombard his patient with powerful antiarrhythmic drugs. Lignocaine, intravenously in $100-\mathrm{mg}$ doses, has an antiarrhythmic effect lasting for some 20 minutes and is probably the drug of choice. Procaineamide and phenytoin sodium are other antiarrhythmic drugs of value for treating apparently lignocaine-resistent arrhythmias.

Sinus or nodal bradycardia may allow an unstable rhythm to develop, with the danger of ventricular fibrillation." Intravenous atropine in an initial dose of $0.3 \mathrm{mg}$, may be repeated, until the heart rate is increased to around 70-80 per minute, thus stabilizing the rhythm and improving the haemodynamic situation.

Unfortunately it is our experience that cardiac arrest, as often as not, occurs without adequate warning and therefore the best form of treatment would be a routinely administered antiarrhythmic agent. Oral procaineamide has been shown effective in reducing the incidence of ventricular tachycardia and fibrillation, provided the blood levels can be carefully controlled $^{22}$ - a requirement which places the therapy beyond the reach of many hospitals. A trial of routine lignocaine infusion, admittedly over a low dose range, failed to produce any reduction in the incidence of ventricular arrhythmias. ${ }^{23}$ Therefore, at present it is impossible to answer the frequently raised question about the advisability of injecting lignocaine in general practice or in the casualty department as soon as infarction is diagnosed. 


\section{Supraventricular Tachycardia}

Atrial fibrillation may result in rapid haemodynamic deterioration and require electrical cardioversion under general anaesthesia. The rhythm is so frequently recurrent, however, that it is preferable to control it with digitalis, where the patient's clinical state is not too seriously compromised. ${ }^{24}$ Frequent atrial premature beats normally precede the onset of fibrillation and are an indication for digitalis.

Paroxysmal atrial or nodal tachycardia may be arrested by carotid sinus massage. If not, the subsequent choice of therapy lies between cardioversion with low energy discharge or digitalization, depending again on the degree of clinical deterioration. The cardioselective beta blocking agent, practolol, given intravenously in doses of $5-15 \mathrm{mg}$ is useful for correcting these arrhythmias.

\section{Complete Atrioventricular Block}

This complication arises in $6-8 \%$ of patients with acute infarction and is associated with an increase in mortality. Much depends on the site of infarction. Inferior lesions may produce local ischaemia in the region of the atrioventricular node. A junctional pacemaker then often arises, with a narrow QRS configuration, and provides an adequate heart rate of, say, 50-60 per minute. Anterior infarction, on the other hand, produces complete block by extensive damage to the intraventricular septum and the branches of the bundle of His. In this situation ventricular complexes are broad and slow, and the ventricular pacemaker is unreliable.

Pacing is most likely to be of benefit when the rate is slow, particularly if there is evidence of pulmonary oedema or ventricular irritability. The pacing catheter is most easily passed from an antecubital vein into the apex of the right ventricle. The catheter tip must be adjusted until pacing is achieved with a stimulus of 1 volt or less. All equipment used must be properly earthed. A demand or standby pacemaker must be used because the block is usually temporary and on return of normal conduction there will be potentially hazardous competition between the physiological pacemaker and its artificial counterpart, with the danger of ventricular fibrillation if a fixed rate device is employed. After normal conduction has returned the catheter should be left in situ for a further four or five days, since recurrence of block is not uncommon.

The development of complete atrioventricular block must always be anticipated in those patients showing complete bundle-branch block, but two forms of conduction defect are particularly sinister. Firstly, right bundle branch block with left axis deviation, and, secondly, occasional dropped beats with a normal P-R interval (Mobitz Type II block). ${ }^{25}$ Both E.C.G. patterns are an indication for the insertion of a pacemaker catheter in anticipation of complete atrioventricular block, which may be sudden in onset and possibly fatal if a ventricular pacemaker fails to emerge.

A recent review of pacing in complete atrioventricular block complicating acute myocardial infarction suggests that it may reduce the overall mortality of this complication from around $50-58 \%$ to $47 \%{ }^{26} \mathrm{My}$ experience of 78 patients, of whom 66 were paced with a mortality of $40 \%$, would suggest that this assessment may be slightly pessimistic. An alternative treatment is intravenous isoprenaline. This will certainly increase the ventricular rate in the presence of complete block, but infusions are difficult to control accurately for several days and this form of treatment should be used only as a temporary expedient while preparing for pacing.

Permanent block after infarction may be more common than is generally recognized. In those patients in whom a return of sinus rhythm is accompanied by the pattern of right bundle-branch block and left axis deviation complete atrioventricular block is likely to arise at some future date.

\section{Left Ventricular Failure}

Together with shock the complication of myocardial failure remains a major cause of death after infarction. Digitalis should not be withheld and together with parenteral diuretic therapy and oxygen forms the cornerstone of treatment. Occasionally pulmonary oedema is so copious as to well out of the patient's mouth and he is literally drowning in oedema fluid. Rapid endotracheal intubation, aspiration of oedema fluid, and positive pressure ventilation may under these circumstances be life saving.

\section{Shock}

The term shock should be restricted to that state of severe hypotension, oliguria, mental confusion, and poor peripheral perfusion, together with a metabolic acidosis which persists in spite of conventional treatment with analgesics, oxygen, and raising of the feet. The mortality is extremely high among these patients and no form of therapy has been found to be uniformly effective, possibly because the clinical picture conceals a large variety of haemodynamic situations. Probably the condition is secondary to massive myocardial injury, ${ }^{27}$ though the persistence of hypotension and hypoxaemia may lead to further extension of the infarct.

Treatment with pressor amines has fallen from favour since the rise of left ventricular systolic pressure which they cause is known to be associated with an increased oxygen requirement of the myocardium. Therapy with vasodilators, with the object of improving peripheral blood flow, is still in the experimental phase. A haemodynamic state has been described in which shock and a low left atrial pressure coexist. $^{28} 29$ Clinical and haemodynamic improvements follow expansion of the circulating volume. The frequency of this situation in shock remains to be assessed; we have measured the pulmonary wedge pressure in seven patients in cardiogenic shock and have always found it to be raised.

Of the many forms of mechanical assistance devised in recent years the most hopeful appears to be that of aortic balloon counterpulsation. A non-occluding balloon is introduced into the aorta from the femoral artery and is inflated in phase with the cardiac cycle. Inflation occurs during the period of ventricular diastole and deflation in ventricular systole. The aim is to reduce ventricular systolic pressure, and hence the myocardial oxygen requirement, and to increase diastolic blood pressure, and thereby augment coronary blood flow. ${ }^{30}$

It has recently been claimed that early diagnosis of infarction coupled with arrhythmic control may dramatically reduce the incidence of shock. ${ }^{31}$ Herein lies the patient's main hope, for our existing methods for the treatment of shock are still very unsatisfactory. 